ein Gemenge von Betain- und Cholinchlorid in Händen gehabt hat, denn das Betainchlorid ist wenig hygroskopisch und verlangt nur $23,1 \% \mathrm{Cl}$, würde also den Chlorwert drücken, wie es bei der Substanz Görte's geschehen ist. Görte konnte denn auch durch die etwas verschiedene Löslichkeit der Platinate beide Substanzen trennen. Sicher hätte ein so guter Untersucher wie Görte schon vor mir das Betain im Champignonextrakt aufgefunden, wenn er mehr Material gehabt hätte.

Das Filtrat von den Betain- und Cholinquecksilberverbindungen versetzte ich noch mit alkoholischer Natriumacetatlösung. Es fiel ein geringer Niederschlag aus zum Zeichen, daß sich nur noch geringe Massen basischer Bestandteile der Aufteilung entzogen hatten, und bestätigt dieser Befund die Angabe Görte's, nach der sich in den von ihm untersuchten Hutpilzen nur kleine Meagen organischer Basen befinden. Die hauptsächlichste Base ist leider eine anorganische, nämlich das Kalium.

Bezüglich der physiologischen Wirkung sind wir über die des Kaliums und Cholins ziemlich gut unterrichtet. Sie sind ja auch die an Menge hervorragenden, aber selbst sie sind wahrscheinlich nicht reichlich genug im Champignonextrakt vorhanden, um bei mäBiger Verdünnung desselben, vom Magen-Darmkanal genommen, die ihnen spezifische Tätigkeit zu entfalten. Es müssen demnach Substanzen sein, die durch Phosphorwolframsäure nicht fällbar sind, welche die von den Klinikern beobachtete günstige Beeinflussung des Kranken zu vollziehen vermögen.

\title{
Über das auf Alkali-Einwirkung beruhende Verfahren der polarimetrischen Zuckerbestimmung nach Jolles.
}

\author{
Von \\ Dr. Bruno Bardach und Dr. Siegmund Silberstein. \\ Mitteilung ans dem Chemisch-mikroskopischen Laboratorium von \\ Dr. Bruno Bardach und Siegmand Silberstein in Wien. \\ [Eingegangen am 20. Februar 1911.]
}

Die Einwirkung von Alkali anf das optische Drehungsvermögen von Zucker-

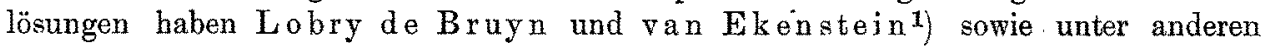
auch A. Jolles ${ }^{2}$ ) untersucht. Letzterer hat ein auf diesem Verhalten beruhendes Verfahren zur quantitativen Bestimmung der Saccharose neben anderen Zuckerarten empfohlen ${ }^{3}$ ). Die Einwirkung des Alkali erfolgt nach Jolles auf drei Arten und

1) Recueil des travaux chimiques des Pays Bas, 14, 156, 203; 16, 262; Ber. Deutsch. Chem. Gesellsch. 1895, 28, 3078; Zeitschr. Deutsch. Zuckerindustrie 1908, 45, 949, 1090; 1910, 47, 1026.

2) Biochem. Zeitschr. 1910 (3), 29. 152.

3) Diese Zeitschrift 1910, 20,631. 
zwar durch 3/4-stündiges Erhitzen im kochenden Wasserbade am Rückflußkühler oder durch $3 / 4$-stündiges Erhitzen im Lintner'sehen Druckfläzchchen im kochenden Wasserbade oder schlieblich durch 24 -stündiges Erwärmen bei $37^{0}$ im geschlossenen Fläschchen im Thermostaten. Um das Verfahren zu erproben, stellten wir mehrere Versuche mit reinen Glykoselösungen (Kahlbaum) sowie Gemischen dieser mit Saccharoselösuugen (Würfelzucker) unter Anwendung von 1/10 N.-Alkalilauge an. Durch diese orientierenden Versuche waren wir zu dem Ergebnisse gekommen, daf alle drei Verfahren bei möglichst genauer Einhaltung der Vorschrift verwendbare Ergebnisse liefern können. So erhielten wir im Thermostaten bei einer 1 und $2 \%$-igen G!ykoselösung Polarisationswerte von - 0,02 bezw. - 0,050; die Rechtsdrehung war also praktisch zum Verschwinden gebracht worden.

Da durch das Erhitzen bei höherer Temperatur - namentlich im Lintner'schen Fläschen - durch Zersetzung allzu dunkel gefärbte Lösungen entstanden, die beim Polarisieren gewisse Schwierigkeiten boten, im Thermostaten dagegen relativ hell gefärbte Flüssigkeiten erhalten wurden, haben wir bei weiterer Durchführung dieser Untersuchungen das Hauptgewicht auf Versuche bei niederer Temperatur, das ist im Thermostaten gelegt. Hierbei haben wir die Wahrnehmung gemacht, daß die Einwirkung bei Luftzutritt die Abnahme des Drehungsvermögens beschleunigt und gleichzeitig eine schwächere Verfärbung hervorruft. Die hellere Beschaffenheit der so behandelten Lösungen ließ ein direktes Polarisieren im $200 \mathrm{~mm}$-Rohr ohne weitere Verdünnungen oder Zusätze (Bleiessig etc.) zu, sodass die hierdurch sich ergebende Fehlerquelle vermieden werden konnte. Ferner haben wir zur Verminderung der infolge von Verdünnungen sich ergebenden Rechnungsfehler auch möglichst konzentrierte Lösungen verarbeitet. Nach dem so abgeänderten Verfahren wurden die Versuche, wie folgt, angestellt:

Fine gemessene Menge der zu untersuchenden Zuckerlösung wird mit soviel N.-Natronlauge versetzt, daß die Lösung 1/10 $\mathrm{n}$.-alkalisch wird. Am einfachsten füllt man genau gemessene ${ }^{1}$ ) $45 \mathrm{ccm}$ der neutralen Zuckerlösung mit N.-Natronlauge im $50 \mathrm{ccm}-\mathrm{Kölbchen}$ auf. Die Lösung wird polarisiert und eine gemessene Menge in ein Becherglas (8-10 cm Höhe und etwa $5 \mathrm{~cm}$ innerer Durchmesser) gebracht und nun im Thermostaten 20 Stunden $^{2}$ ) (ohne Unterbrechung) bei einer Durchschnittstemperatur von etwa $37^{1} / 2^{0}\left(36-39^{0}\right)$ offen stehen gelassen. Nach dieser Zeit werden die Bechergläser herausgenommen, die Flüssigkeit wird bei Zimmertemperatur abkühlen gelassen, wieder auf das Volumen vor der Polarisation mit Wasser unter Nachwaschen aufgefüllt und neuerdings polarisiert.

So behandelte Lösungen ergaben bei der Polarisation folgende Werte:

1) Bei konzentrierter Saccharoselösungen sind an Stelle der Messungen, Wägungen za setzen.

2) Die Festsetzung der Einwirkungsdauer erfolgte auf Grund der Wahrnehmung, dak bei Einhaltung von 20 Stunden in offenen Gefaßen die Einwirkung des Alkalis auf Glykoselösungen meist nicht nur die Polarisation ganz aufhob, sondern daf sich sogar noch schwache Linksdrehungen ergaben. Die Erwärmung hat ohne Unterbrechung zu erfolgen, da merkwürdigerweise bei der einmal abgekühlten Probe der Polarisationswert bei nenerlichem Erwärmen nur unvollkommen abnimmt. 
Glykoselösungen.

\begin{tabular}{|c|c|c|c|}
\hline \multirow{2}{*}{ No. } & \multirow{2}{*}{$\begin{array}{l}\text { Ungefährer Gehalt der } \\
\text { Lösung an Glykose }\end{array}$} & \multicolumn{2}{|c|}{ Polarisationen im $200 \mathrm{~mm}$-Kohr (Kreisgrade Wild) } \\
\hline & & Vor der Einwirkung & Na ch der Einwirkung \\
\hline 1 & 0,50 & $+0,51$ & $-0,09$ \\
\hline 2 & 1 & $+1,02$ & $-0,19$ \\
\hline 3 & 1 & $+1,02$ & $-0,15$ \\
\hline 4 & 1,5 & $+1,53$ & $-0,26$ \\
\hline 5 & & $+2,04$ & $-0,25$ \\
\hline 6 & $2 "$ & $+2,05$ & $-0,26$ \\
\hline 7 & 2,5, & $+2,54$ & $-0,36$ \\
\hline 8 & & $+3,05$ & $-0,26$ \\
\hline 9 & 3 & $+3,06$ & $-0,27$ \\
\hline 10 & 4 & $+4,10$ & $-0,32$ \\
\hline 11 & 4 & $+4,07$ & $-0,25$ \\
\hline 12 & 5 & $\begin{array}{r}+5,12 \\
\end{array}$ & $-0,21$ \\
\hline
\end{tabular}

Es traten somit bei Glykoselösungen von verschiedenem Gehalt $\left(0,5-5^{0} \%\right)$ nach 20-stündigem Erwärmen stets ziemlich gleiche Linksdrehungen ${ }^{x}$ ) auf, sodaß wir es vorzogen, als Ende der Reaktion nicht den Wert $0^{0}$, sondern eben den empirisch festgestellten Wert von etwa $-0,25^{\circ}$ anzunehmen.

Darnach berechnet man in Glykose-Saccharose-Gemischen den Polarisationswert der Glykose (D) nach der Formel: $\mathrm{D}=\mathrm{A}-\mathrm{B}-0,25$ und dementsprechend den Polarisationswert der Saccharose (S) nach der Formel: $\mathrm{S}=\mathrm{B}+0,25$, wobei $\mathrm{A}$ der Polarisationswert vor der Einwirkung, B derjenige nach der Einwirkung ist.

Die nachstehende Tabelle enthält eine Anzahl Glykose-SaccharoseBestimmungen.

\begin{tabular}{|c|c|c|c|c|c|c|c|c|}
\hline \multirow{2}{*}{ No. } & \multicolumn{2}{|c|}{$\begin{array}{l}\text { ZusammensetzungderLö- } \\
\text { sungen. Zu } 5 \text { cem N.-Natron- } \\
\text { lange wurden zugesetzt: }\end{array}$} & \multicolumn{2}{|c|}{$\begin{array}{l}\text { Polarisation im } 200 \mathrm{~mm} \text { - } \\
\text { Rollr (Kreisgrade Wild) }\end{array}$} & \multicolumn{2}{|c|}{$\begin{array}{c}\text { Polurisationswerte de: } \\
\text { Glykose }\end{array}$} & \multicolumn{2}{|c|}{$\begin{array}{l}\text { Polarisationswerte der } \\
\text { Saecharose }\end{array}$} \\
\hline & $\begin{array}{c}\text { von etwa } 10 \% \\
\text { iger Glykose- } \\
\text { lösung }\end{array}$ & $\begin{array}{l}\text { von etwa } 10 \%- \\
\text { iger Saccha- } \\
\text { roselösung }\end{array}$ & $\underset{\text { wirk }}{\text { vor de }}$ & $\begin{array}{c}\text { nach der } \\
\text { Einwirkung } \\
\text { (B) }\end{array}$ & gefunden & berechnet & gefunden & berechnet \\
\hline 1 & $5 \mathrm{ccm}$ & $40 \mathrm{cem}$ & & & & & & \\
\hline 2 & & & & + & & & & \\
\hline 3 & 15 & & & +7 & & & &, 580 \\
\hline 4 & 20 & & +1 & +6 & & & & $+6,330$ \\
\hline 5 & 25 & 20 & +10 & $+4,91^{0}$ & +5 & & $+5,160$ & $+5,060$ \\
\hline 6 & 5 & 40 & $+11,45^{\prime \prime}$ & $+10,140$ & $+1,06^{\circ}$ & $+1,020$ & $+10,390$ & $+10,430$ \\
\hline 7 & 15 & 30 & $+10,8$ & $+7,46^{0}$ & +3 & $+3,050$ & $+7,710$ & $+7,820$ \\
\hline 8 & 25 & 20 & $+10,310$ & $+4,91^{\circ}$ & $+5,150$ & $+5,100$ & $+5,16^{0}$ & $+5,210$ \\
\hline
\end{tabular}

1) Dieser leicht ziemlich konstant zu erhaltende Wert der Linksdrehung entspricht einfach einer bestimmten Phase des Gleichgewichtszustandes

Glykose $\rightleftarrows$ Hruktose $\rightleftarrows$ Mamose,

welcher allgemein eintritt, wenn sehr verdünnte wässerige Alkalien auf Hexosen einwirken. 
Aus diesen Polarisationswerten geht hervor, daß in Saccharose-Glykose-Gemischen bei 20-stündigem Erwärmen im Thermostaten mit den angegebenen Mengen Alkali beide Zuckerarten bestimmt werden können. Die Glykosebestimmungen stimmen naturgemäß bei konzentrierteren Lösungen $(3-4 \%)$ besser, da die sehr kleinen $A b-$ weichungen von der empirischen Korrektur von $-0,25^{0}$ prozentual eben weniger in Betracht kommen. Zuckermischungen mit einem Glykosegehalt von mehr áls $5 \%$ sind entsprechend zu verdünnen. Die Konzentration der Saccharose kommt nicht in Betracht, nur sind zur Vermeidung von Fehlern die zu untersuchenden Mengen, wie bereits bemerkt, zu wägen statt zu messen. Bleibt der Polarisationswert vor und nach der Einwirkung der gleiche, so ist Glykose eben nicht vorhanden und entfällt somit selbstverständlich auch jede Korrektur.

Bemerkt sei noch, daß ein größerer Saccharosegehalt eine kleine, jedoch die Korrektur nicht berührende Verminderung der Linksdrehung der Glykoselösung hervorruft und eine schwächere Verfärbung der letzteren bewirkt. Ahnliches Verhalten zeigten Zuckerlösungen bis zu einem Gehalt von 2\% Glykose bei Einwirkung von etwas höherer Temperatur. Jedoch lieferten die etwa 18 Minuten in einem Wasserbade von $65-70^{\circ}$ angestellten Versuche weniger gute Ergebnisse wie im Thermostaten und wurden deshalb nicht weiter verfolgt.

$\mathrm{Da}$ sämtliche bisher angeführten Versuche mit reinen wässerigen Zuckerlösungen angestellt worden waren, baben wir, um den Einflu? von Salzen auf die Reaktion zu prüfen, noch einige Versuche unter Zusatz von Salzen eingestellt. Das Verhalten derselben war eigentümlicherweise nicht ganz gleich, indem Chlornatrium $(1 \%)$ ohne Einfluf, Natriumsulfat $(1 \%)$ und Natriumcarbonat $(1 \%)$, allerdings nur bei höherem Glykosegehalt sehr wenig verzögernd auf den Verlauf wirkten, sodab z. B, eine 4\%-ige Glykoselösung nach 20 Stunden noch nicht ganz auf dem empirischen Polarisationswert unter $0^{0}$ angelangt war. Geringe Mengen dieser Salze 1 ) können also hinsichtlich ihrer Wirkung vernachlässigt werden.

Die von uns angeführte Abänderung des Verfahrens gibt somit für Glykose-Saccharose-Mischungen sehr brauchbare Werte.

In welcher Weise andere Zuckerarten zur Erzielung konstanter Werte mit verdünnter Lauge im Thermostaten zu behandeln sind, wäre durch weitere Versuche festzustellen.

1) Wir hatten auch hierbei gärungshemmende Substanzen berücksichtigt. 0,6\% Salicylsäure war nach dem Neutralisieren der Lösung ohne Linfluß, hingegen vermochte Borsäure in 1\%-iger Lösung nach dem Neutralisieren die Einwirkung ganz zu verhindern. 